\title{
3D video representation and design for ubiquitous environments
}

\author{
Xingang Liu $\cdot$ Shu-Ching Chen • Jianhua Ma • \\ Laurence T. Yang
}

Published online: 7 June 2013

(C) Springer Science+Business Media New York 2013

With the fast developments in electronics industry and the amazing growing of the potential customers, multimedia are increasingly ubiquitous: more and more people live in a world of Internet pop-ups and streaming television, mobile phone texting and video clips, MP3 players and pod-casting. Especially, there have been rapid advancements in 3D techniques and technologies. A number of systems have been developed for dynamic 3D reconstruction from multiple view videos over the past decade. Hardware has both improved and become considerably cheaper, making real-time and interactive 3D available to the hobbyist, as well as to the researcher. There have been major studies in such areas as molecular modeling, photogrammetry, flight simulation, $\mathrm{CAD}$, visualization of multidimensional data, medical imaging, tele-operations such as remote vehicle piloting and remote surgery, and stereo lithography. In computer graphics, the improvements in speed, resolution, and economy make interactive stereo an important capability. Old techniques have been improved, and new ones have been developed. True 3D is rapidly becoming an important part of computer graphics, visualization, virtual reality systems, and computer gaming. Numerous 3D systems are granted patents each year, but very few systems move beyond the prototype stage and become commercially viable. Here we treat the salient 3D systems. Therefore, the 3D contents and applications will be the trends of the future multimedia communication. This special issue is intended to provide a forum for presenting, exchanging and discussing the

\footnotetext{
X. Liu $(\bowtie)$

University of Electronic Science and Technology of China, Chengdu, China

e-mail: hanksliu.xg@gmail.com

S.-C. Chen

Florida International University, University Park, FL, USA

e-mail: chens@cs.fiu.edu

J. Ma

Hosei University, Tokyo, Japan

e-mail: jianhua@hosei.ac.jp

L. T. Yang

St. Francis Xavier University, Antigonish, Nova Scotia, Canada

e-mail: 1tyang@gmail.com
} 
3D multimedia communications and recent advances on multimedia technologies for ubiquitous environments. Among submissions that covered a variety of interesting topics, two rounds of careful review by the experts in the field led to 15 papers for inclusion in this issue.

The first paper "A Network Algorithm for 3D/2D IPTV Distribution using WiMAX and WLAN Technologies" by J. Mauri et al. presented a network algorithm manages the IPTV access network and decides which type of wireless (WiMAX and WLAN) technology the customers should connect with when they are using multiband devices in order to receive the best 3D/2D IPTV QoE. This decision is taken based on the requirements of the IPTV client device, the available networks, and some network parameters (such as the number of loss packets and packet delay), to provide the maximum QoE to the customer. The authors describe the algorithm and explain the protocol for the proper operation of their proposal. The measurements taken in a real environment demonstrate the success of their system.

As 3D contents have gained significant popularity and become ubiquitous, ensuring satisfactory quality of experience (QoE) of the $3 \mathrm{D}$ contents has become an important issue. Especially, subjective quality assessment is needed to understand the $3 \mathrm{D}$ quality perception of humans, which is eventually useful for optimization of 3D processing techniques. In order to facilitate reliable subjective quality assessment, J. Lee et al., in the paper entitled by "Paired Comparison-based Subjective Quality Assessment of Stereoscopic Images", proposed a new subjective test methodology based on paired comparison. The complete test procedure, from experiment design to outlier detection and score analysis, is described. Experimental results on a stereoscopic image database with varying camera distances demonstrate the usefulness of the method. The third paper entitled by "Advanced Motion Vector Coding Framework for Multiview Video Sequences" by S. Ryu et al. is related to the topic of 3D video codec in which multiview video plus depth (MVD) format that is the most advanced 3D video format. Even though the MVD format is rate-efficient approach to generate high quality 3D video, bits required for such format are very heavy to be managed in ubiquitous environments. The method proposed in this paper can reduce the bits required for MVD format. As a result, high quality 3D video service is available in ubiquitous environment.

In the paper entitled by "Data Generation and Representation Method for 3D Video Conferencing using Programming by Demonstration" by Y. Sung and K. Cho investigated video conferencing which provides an environment where multiple users can communicate at the same time as a key feature in the ubiquitous environment. They proposed a framework to present a user using a virtual human. By applying programming by demonstration, a system can automatically collect and generate the various data automatically without any prior knowledge of the video conference system. Then, the design of framework to represent users in the 3D Video conferences of ubiquitous environments was introduced. In the paper entitled by "A Real-time Dynamic Key Management for Hierarchical Wireless Multimedia Sensor Network", Y. Zhang et al. pointed out that wireless Multimedia Sensor Networks (WMSNs) are specially designed for retrieving multimedia data, such as, 3D video, images and scalar sensor data, etc. over ubiquitous environments. They presented splay tree-based real-time dynamic key management (RDKM) provides dynamic architecture to generate new keys and makes the dynamic key management feasible without any overhead in the LEACH-like protocol. Thus, even WMSNs are subjected to numerous threats and vulnerable to various attacks, the RDKM can efficiently protect the multimedia streams and address challenging security issues of runtime in WMSNs. In the paper entitled by "Quality-Driven Secure Audio Transmissions in Wireless Multimedia Sensor Networks", H. Wang et. al., proposed an energy-efficient and quality-driven watermarking system is proposed for secure 
audio transmissions in WMSNs. It simultaneously controls the resource allocation at the MAC-PHY layers and the watermark embedding at the application layer to optimize audio quality and assure the authentication performance. The proposed watermarking scheme does not only achieve the robustness and transparency desired in general compression approaches (i.e., MPEG-1, MPEG-2 and MP3), but also it is shown to be resistant to the distortion due to the wireless channel.

In the survey paper entitled by "Classification Algorithms for Interactive Multimedia Services: A Review" by C. Tsai et al. extensively surveyed various remarkable techniques for interactive multimedia services. For the ubiquitous media objects, devices, and activities are indispensable to our daily life in the future and interactive multimedia services are an inevitable outcome of the ubiquitous environment, this survey presented an overview of the clustering and classification algorithms, which can be used to improve the performance of interactive multimedia systems. The authors begin with a brief introduction to interactive multimedia systems and continue with a review of the clustering and classification algorithms for interactive ubiquitous multimedia systems, which consists of concepts, research directions, and applications of these algorithms. Additionally, M. Chen in "Towards Smart City: M2M Communications with Software Agent Intelligence" focuses on recent advances in the fields of wireless technology which have exhibited a strong potential and tendency on improving human life by means of ubiquitous communications devices that enable smart, distributed services. A four-layer architecture for internet of things (IoT) was proposed. Based on the architecture, M. Chen further presented a novel intelligent system for machine to machine communications.

Context-awareness assists 3D video applications to discover the changeable contextual information and adapting their behaviors accordingly in ubiquitous environments. In the paper "Survey on context-awareness in ubiquitous media", D. Zhang et. al. proposed a reference framework to identify key functionalities of contextawareness, facilitating designing and developing context-aware 3D video applications in ubiquitous media. In the paper "QoS Provisioning Wireless Multimedia Transmission over Cognitive Radio Networks" by Y. Ge et al., addressed a new challenging issue regarding how to satisfy the QoS requirements of wireless multimedia transmission over Cognitive Radio networks, considering the limited spectrum resources have become more and more scarce and they are in an inefficiency usage by a fixed spectrum assignment policy. In the paper "A Two Step Salient Objects Extraction Framework based on Image Segmentation and Saliency Detection" by Q. Liu et al., the salient objects extraction framework is divided into two steps. First, the image is segmented into several regions using image segmentation algorithm and the saliency map for the whole image is detected with saliency detection algorithm. Then, for each region, some features are extracted to classify the region as a background region or a salient region twice. In the paper entitled by "Design and Application of the Stereo Vision Manipulator with Novel Scheduling Policies Control", K. Hsu et al. aim at the establishment of a highly efficient and low-cost vision distance measuring system which is steady, cheap, and suitable for application in machine vision. In the paper "Enhanced MAC Protocol to Support Multimedia Traffic in Cognitive Wireless Mesh Networks", R. Zhu et al. an efficient CSMA/CA compatible TDMA-like MAC protocol (T-MAC) and an optimal adaptive scheduling scheme are proposed to support ubiquitous media transmission, which guarantee quality of service of different priority real-time multimedia traffic with allocating more channel access time in centralized manner and seeking the schedule among all possible sequences of concurrent transmissions to minimize the occupied resources. The results show the proposed methods 
are efficient multimedia applications transmission schemes for mobile terminals in cognitive wireless mesh networks. In the paper "Inference Topology of Distributed Camera Networks with Multiple Cameras", Y. Nam et al. presented an intelligent video surveillance system with the metadata rule to exchange analyzed information between distributed and heterogeneous surveillance systems. The intelligent surveillance system provides various services that are object identification, object size analysis, object localization, tampering detection, activity recognition, and moving object tracking. A 3- tier context-awareness conceptual framework is presented to identify the design principles of the intelligent surveillance system. The design framework and the implementation of the prototypes have served as a logical basis to elaborate broad design concepts and intelligent video computing technologies that will be performed toward future smart surveillance systems.

In the last paper entitled by "Fast Moving Object Detection with Non-stationary Background", J. Kim proposed a novel moving objects detection algorithm for free-moving cameras. By incorporating the optical flow clustering and Delaunay triangulation algorithm, our algorithm is especially suitable when building video surveillance systems using free moving cameras for ubiquitous environment. The experimental results on CMU database demonstrate that our algorithm outperforms the existing other methods in terms of detection accuracy and processing time.

We would like to thank all the reviewers for their efforts and constructive comments. We would in particular like to thank Professor Borko Furht, the Editor-in-Chief, for his support and helpful suggestions during the very delicate stages of concluding the special issue. Finally, we would like to thank all the authors who submitted their precious research work to this special issue.

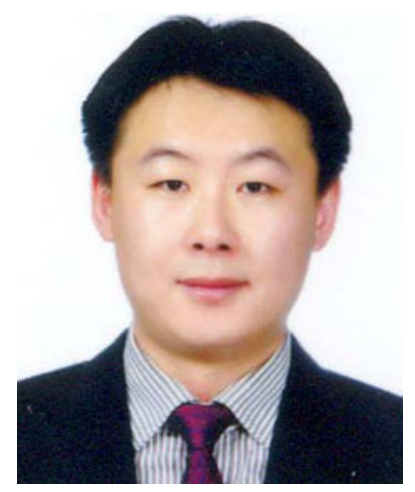

Xingang Liu Dr. Liu is currently the associate professor in University of Electronic Science and Technology of China (UESTC). He received the B.S. degree in the school of electronic engineering (EE) in UESTC in 2000, and the M.S. and Ph.D. degrees both in Yeungnam University of South Korea in 2005 and 2010, respectively. During 2000 to 2003, he was a faculty member in EE of UESTC. He worked as BK21 research fellow in the school of Electrical and Electronic Engineering in Yonsei University of South Korea during 2010 to 2011. His research interests are multimedia signal communication related topics, such as heterogeneous and homogenous video transcoding, video quality measurement (QoErelated), video signal error concealment in the destination, mode decision algorithm, 3D video codec and so on. He has published around 50 academic papers in refereed journals, conference proceedings as the first or corresponding author. Dr. Liu is a member of IEEE, KSII and KICS. He has been invited to serve as an organization committee, technical program committee and session chair of around 20 IEEE International conferences/workshops/symposiums. 


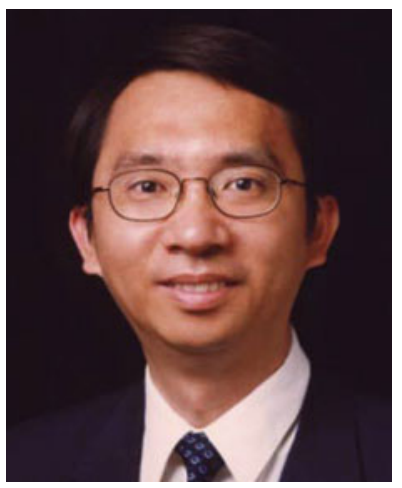

Shu-Ching Chen Dr. Shu-Ching Chen is a Full Professor in the School of Computing and Information Sciences (SCIS), Florida International University (FIU), Miami since August 2009. Prior to that, he was an Assistant/Associate Professor in SCIS at FIU from 1999. He received his Ph.D. degree in Electrical and Computer Engineering in 1998, and Master's degrees in Computer Science, Electrical Engineering, and Civil Engineering in 1992, 1995, and 1996, respectively, all from Purdue University of USA. He is the Director of Distributed Multimedia Information Systems Laboratory and Associate Director of the Center for Advanced Distributed System Engineering at SCIS. His main research interests include content-based image/video retrieval, distributed multimedia database management systems, multimedia data mining, multimedia systems, and Disaster Information Management. Dr. Chen has authored and coauthored more than 250 research papers in journals, refereed conference/symposium/workshop proceedings, book chapters, and three books. He is the founding Editor-in-Chief of International Journal of Multimedia Data Engineering and Management and Associate Editors/Editorial Board for other 15 journals. He is the Chair of IEEE Computer Society Technical Committee on Multimedia Computing and Co-Chair of IEEE Systems, Man, and Cybernetics Society's Technical Committee on Knowledge Acquisition in Intelligent Systems.

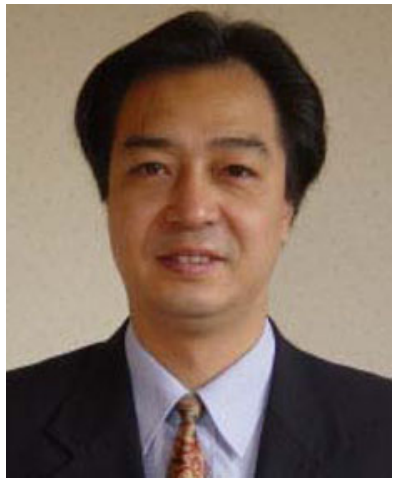

Jianhua Ma Dr. Ma is a member of IEEE and ACM. He has edited over 15 books/proceedings, and published more than 200 academic papers in journals, books and conference proceedings. He has delivered about 20 keynote speeches in international conferences, and given invited talks in over 40 universities/institutes. He received the Best Paper Award from the 2000 International Conference on Information Society in the 21st Century: Emerging Technologies and New Challenges, and the Highly Commended Paper Award from the 2004 IEEE International Conference on Advanced Information Networking and Applications. He is the Chair of IEEE Task Force on Autonomic and Trusted Computing, a co-founder of IEEE CIS Task Force on Ubiquitous Intelligence and Computing, and received the Certificate of Appreciation from IEEE Computer Society in 2004-2007. 


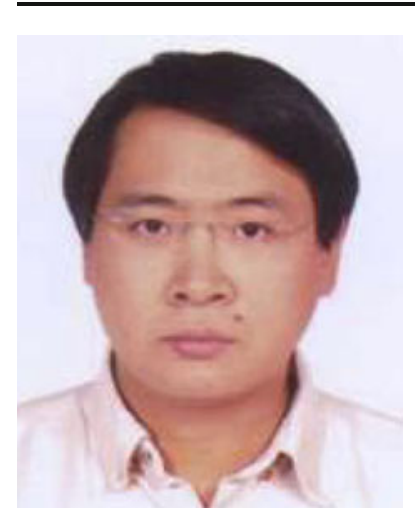

Laurence T. Yang Prof. Laurence T. Yang graduated from Tsinghua University, China and got his Ph.D. in Computer Science from University of Victoria, Canada. He joined St. Francis Xavier University in 1999. His current research includes parallel and distributed computing, embedded and ubiquitous/pervasive computing. He has published many papers in various refereed journals, conference proceedings and book chapters in these areas. He has been involved actively in conferences and workshops as a program/general/steering conference chair and numerous conference and workshops as a program committee member. In addition, he is the editorsin-chief of several international journals. He is serving as an editor for many international journals. He has been acting as an author/co-author or an editor/co-editor of many books from Kluwer, Springer, Nova Science, American Scientific Publishers and John Wiley \& Sons. He has been invited to give around 20 keynote talks at various international conferences and symposia. 